\title{
Interfaces
}

INTERFACES Image Texte Language

$46 \mid 2021$

Jeux de Formats (2)

\section{«L'architecte doit être le metteur en œuvre du monde » : format et métamodèle chez Peter} Greenaway

Lawrence Gasquet

\section{CpenEdition}

Journals

Édition électronique

URL : https://journals.openedition.org/interfaces/3669

DOI : 10.4000/interfaces.3669

ISSN : 2647-6754

Éditeur :

Université de Bourgogne, Université de Paris, College of the Holy Cross

\section{Référence électronique}

Lawrence Gasquet, « "L'architecte doit être le metteur en œuvre du monde » : format et métamodèle chez Peter Greenaway », Interfaces [En ligne], 46 | 2021, mis en ligne le 15 décembre 2021, consulté le 22 juin 2022. URL : http://journals.openedition.org/interfaces/3669 ; DOI : https://doi.org/10.4000/ interfaces.3669

Ce document a été généré automatiquement le 22 juin 2022.

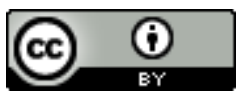

Les contenus de la revue Interfaces sont mis à disposition selon les termes de la Licence Creative Commons Attribution 4.0 International. 


\title{
«L'architecte doit être le metteur en œuvre du monde » : format et métamodèle chez Peter Greenaway
}

\author{
Lawrence Gasquet
}

Kracklite : What's the scale? Frederico : It's what you asked for. Kracklite : In centimetres or inches? Frederico : Centimetres! No self-respecting architect uses inches.

Io : Did Boullée use inches? Julio (with a laugh): He used Boullées. Peter Greenaway, The Belly of An Architect

Une forme sans son support n'est pas forme, et le support est forme lui-même. Henri Focillon, Vie des formes

L'histoire de toute forme d'art connaît des époques critiques où cette forme aspire à des effets qui ne peuvent s'obtenir sans contrainte qu'à base d'un standard technique transformé, c'est-à-dire dans une forme d'art nouvelle. Les extravagances et les crudités de l'art, qui se produisent ainsi particulièrement dans les soidisant époques décadentes, surgissent en réalité de son foyer créateur le plus riche. Walter Benjamin, L'Euvre d'art à l'époque de sa reproductibilité technique

Prenant le contre-pied de la célèbre formule d'Alberti qui en 1435 proposa d'utiliser le cadre d'une fenêtre afin de représenter le monde depuis le point de vue fixe d'un 
observateur, ${ }^{1}$ Peter Greenaway refuse de se soumettre entièrement à la dictature de ce premier cadre (qui était à l'origine pour Alberti un simple instrument, avant que l'art occidental ne l'institue en format) lorsqu'il déclare que son travail n'est justement pas une fenêtre qui s'ouvre sur le monde : " [My work is not] a window on the world, not a door out onto the next-door neighbour's garden, it is a self-conscious and artificial construct " (Greenaway, in Elliott \& Purdy, 122). La représentation perspectiviste s'effectue par le biais d'un cadre-limite qui contre toute attente ouvre le champ, et pose l'illusion de la profondeur comme convention, définissant ainsi le format standardisé de la représentation picturale en Occident après la Renaissance. La notion de format fait jaillir de nombreuses questions, et travaille le champ de l'art de manière constante, voire violente, depuis des siècles. Comme le remarque David Zerbib, la notion de format renvoie à un plan mal identifié, " parce que trop relégué ou au contraire trop exposé dans l'évidence d'une variable technique ou d'une mesure quantitative. Il semble pourtant légitime de le considérer comme un opérateur clé des pratiques matérielles et symboliques [...] Nous proposons de l'entendre [...] comme construction médiatrice qui norme des modes d'inscription, de codage, de traduction, d'implémentation, d'exposition, de filtrage et d'usage de formes et d'informations » (Zerbib, 16). Le format et ses avatars sont un des thèmes de prédilection de Peter Greenaway. De manière paradoxale, selon lui, la meilleure façon d'échapper aux pièges de la représentation réaliste est de rendre l'oeuvre aussi "artificielle" que possible, en soulignant subtilement sa facticité. Ainsi, le format selon lui gagne toujours à être souligné, à être reconnu, voire à être dénoncé pour ce qu'il est : un cadre (au sens large) a posteriori de la perception, paradoxalement confondu au fil du temps avec un cadre a priori. «Perhaps it all started with the invention of the artificial frame passed on from painting to theatre to photograph to cinema to television [...]» (Greenaway, in Elliott \& Purdy, 123). L'effet principal du format est de mettre en scène des compatibilités, mais également de créer parfois des incompatibilités, soulignant l'arbitraire des systèmes de signes que nous fabriquons, et au sein desquels nous évoluons en tant qu'homo artifex.

Plasticien et cinéaste, Greenaway analyse minutieusement l'acte de représenter, étudie sa mise en œuvre quant à la manière dont nous découpons et percevons ce qui nous entoure, souvent à l'aide d'outils perceptuels et cognitifs que nous nommons "dispositifs " ; ainsi, les formats normés se sont succédé au fil des siècles et ont façonné notre perception du monde, jusqu'à souvent devenir imperceptibles, et se confondre avec ce dernier. La question du format, à la fois contrainte et condition du voir et du percevoir, obsède Greenaway, et fait l'objet d'une représentation métaréflexive dans son oeuvre. La critique structuraliste s'est longuement penchée sur ses productions, au point d'en faire un de ses hérauts (cf. Willoquet-Maricondi \& AlemanyGalway, Keesey, Woods) car le plasticien éprouve constamment la rigidité des systèmes de signes visant à rendre le monde intelligible. Un des topoï de ses productions est que l'homme doit créer des systèmes de classification efficaces s'il veut parvenir à garder l'ascendant sur le monde. Le réel est perçu comme polymorphe, foisonnant, désordonné, et si l'homme veut le comprendre, et ne pas se laisser engloutir par le chaos, alors il doit trier, simplifier, classer, ordonner. Ne pas le faire condamne à l'ignorance et à la mort, comme l'expérimentent tragiquement quasiment tous les personnages principaux de ses longs-métrages.

3 Les productions de Peter Greenaway, qu'elles soient cinématographiques, sculpturales, picturales, ou textuelles, cherchent toutes à imposer un ordre à un ensemble 
hétéroclite. Si l'on prend les longs-métrages, cet ordre peut être mathématique (Drowning by Numbers), pictural (The Draughtsman's Contract), religieux (Baby of Mâcon), linguistique (Prospero's Books, The Pillow Book), architectural (The Belly of an Architect); la dimension visuelle est omniprésente, et le format se place au centre des préoccupations théoriques de Greenaway. Je m'attacherai ici à étudier l'instauration du format dans les deux œuvres complémentaires que sont le long-métrage The Belly of An Architect (1988) et l'exposition The Stairs (1994); le format sera ici analysé dans un contexte principalement architectural. Tout d'abord, il sera question de décor, ou comment l'architecture transforme l'espace en théâtre; ensuite, nous étudierons comment le format autorise un passage du sensible vers l'intelligible, avant de nous pencher sur les limites imposées au spectateur. En dernière analyse, nous verrons comment le format discipline la perception et autorise l'intellect à se saisir du réel.

\section{L'Architecture ou la dramatisation de l'espace}

4 L'architecture revêt une fonction particulière pour le plasticien britannique, car il la classe parmi les dispositifs variés que nous utilisons lorsque nous découpons le monde afin de le rendre intelligible (cadre, photographie, dispositifs stéréoscopiques, instruments de vision divers, jusqu'au traitement de la lumière elle-même). L'usage que Greenaway fait du dispositif corrobore le fait que c'est le mode de vision ou de lecture d'une œuvre plastique ou littéraire qui lui donne son statut. Cette hypothèse sousentend donc que la forme ne peut exister sans format. La pratique de Greenaway tend à confirmer la théorie critique qui nie la possibilité d'une forme purement esthétique, qui serait détachée des codes culturels ou conventions établies. Comme le souligne David Zerbib, « Il n'y a pas de hors format ; c'est vers cet enjeu que l'accent de l'art s'est déplacé » (354).

5 A la question que lui pose Suzanna Turman en 1992 afin de savoir s'il est plus intéressé par la forme ou le format que par le contenu d'une œuvre, Greenaway répond qu'il existe un paradoxe particulièrement mal accepté en matière d'art, qui est que toute production culturelle voit invariablement son contenu s'atrophier : « Let me make one statement, which people have a lot of difficulty understanding. I sincerely believe that in all cultural activity, content atrophies very rapidly, and all you're left with is form and strategy. Then there is a way in which the form and the strategy themselves become the content» (Greenaway, in Gras, 152). Ceci signifie que le savoir symbolique lié à l'artefact à une époque donnée (par exemple celle de sa conception et fabrication) est condamné à se dissoudre, et qu'en dernier lieu ne restera plus que l'artefact lui-même, résistant au temps de par sa forme. Nous perdons le sens, mais la forme survit et nous émeut tout de même, même si les raisons de l'émotion changent selon les époques. Toute forme se mue ainsi naturellement en un format. C'est le propre de l'œuvre d'art que d'instaurer de nouveaux formats, et plus généralement de tout signe.

6 Cette mutation et labilité des formats autorisèrent par exemple la peinture italienne à devenir le format qui donna naissance, par calque, à l'esthétique du jardin européen : ce passage d'une esthétique de l'illusion de la surface au paysage tridimensionnel façonna la conception que l'homme avait de la nature. ${ }^{3}$ C'est l'analyse méticuleuse des caractéristiques formelles de cette nature sculptée par l'homme qui incita le jeune Greenaway à s'intéresser à la peinture et à l'architecture, par capillarité : «Among the artificial lakes, obelisks in groves, seashell grottoes, ha-has and forcibly removed 
villages, I discovered Capability Brown and Humphrey Repton and realised both of them had learnt everything then practiced from Palladio and Poussin. » (Greenaway, 2010, 38-39). Le jeune homme, élevé à la campagne, découvre ainsi que le paysage est une création humaine, un fragment de culture, une étendue géographique que l'homme transforme longuement pour en faire un signe, une représentation codée, un microcosme reflétant sa propre conception du cosmos depuis la Renaissance. Un signe en général, et un symbole en particulier, déchiffré par un observateur, qui réfère à d'autres éléments du monde ou de sa représentation dans des relations d'enchâssement complexes et infinies. En anglais, l'art de paysager l'espace se nomme landscape architecture; Greenaway comprend alors que le paysage est une construction complexe qui fonctionne comme projection symbolique; en d'autres mots, il agit comme un dispositif.

Greenaway découvre ensuite l'architecture alors qu'il parcourt l'Italie sans le sou, à l'âge de dix-sept ans, et à son retour, ce qui lui plaît tout particulièrement, c'est l'œuvre des architectes qui ne sont pas parvenus à passer à la construction, souvent pour des raisons pécuniaires ou techniques :

After the physical experience of Italy, I tracked and traced it all out in books and paintings back in my London Art School, and learnt about the classical French Italian Etienne-Louis Boullée and enjoyed the paradox that this visionary never built a building. A dilettante architect. It was a justification to dream about imaginary architecture. No need to worry about guttering, toilets and car parks. Since then of course, I have realised that not many architects build buildings and most of them are dilettanti. Look at the way they write. Somnambulated nothingnesses. An ideal situation. To look and walk about and think about architectural spaces without any responsibility whatsoever [...] (Greenaway, 2010, 54).

Cette architecture jamais réalisée à l'échelle 1 est l'exemplification idéale d'un format, au sens où elle définit des conditions d'expérience, trace des cadres de perception, teste des possibilités pratiques; elle articule theoria et praxis, et fonde également des modes de connaissance, de représentation ou de construction du réel. Cette architecture offre de facto un «théâtre parfait » (Greenaway, 2010,59) comme il le précise : «I look for architecture-with-attitude constantly in cinema. And certainly try to have architecture-with-attitude in my own film-making. Many of the feature films I have been associated with have started with a desire to make architecture. » (Greenaway, 2010, 54).

9 Greenaway aime à répéter que s'il est un élément à l'influence duquel on n'échappe jamais, c'est bien l'architecture. On peut, dit-il, ne jamais aller au musée, au cinéma ou au concert de sa vie, mais on ne peut jamais se soustraire à l'architecture. L'architecture est le plus pérenne et le plus démocratique des arts. En outre, écrire sur l'architecture renforce son impact et démultiplie son pouvoir, comme l'avait bien compris Etienne-Louis Boullée qui voyait en elle l'art le plus accessible, de par sa capacité à s'adresser simultanément à toutes classes sociales confondues (c'est ce qu'il nommait «l'architecture parlante »). L'architecture, en sa qualité suprême de mère de tous les arts selon Diderot, emprunte aux arts figuratifs et impartit une force d'émotion toute particulière aux bâtiments. Comme l'écrivait Boullée, "Oui, je le crois, nos édifices, surtout les édifices publics, devraient être, en quelque façon, des poèmes. Les images qu'ils offrent à nos sens devraient exciter en nous des sentiments analogues à l'usage auquel ces édifices sont consacrés. » (1768, introduction). Ut pictura poesis, Ut Pictura Architectura. 


\section{Modélisation théorique et pragmatique}

10 Greenaway se remémore les pérégrinations romaines de sa jeunesse :

I spent seven weeks in Rome when I was seventeen. I had no money, so I walked everywhere, and I scarcely from that time remembered the painting, but I certainly remembered the architecture, and better still how it fitted together - a flow of streets and piazzas, steps and stairs, vistas, cuts and drops, walls and blackages, arches, pillars, columns, panoramas, blind-alleys, steep embankments, peep-holes and sharp corners, blinding white marble, culsde-sacs, cliffs and troughs and hollows, elevations and insides meeting outsides in every possible combination. Liminality. The threshold, the doorway and the lintel. Again good training with which eventually to examine the ambiguity of the image and the ubiquity of the film-frame which some say arose from the view from any window. (Greenaway, 2010, 53)

11 Cette expérience de la liminalité urbaine va de pair avec la sensation corporelle procurée par la marche; l'homme s'approprie l'espace par le sens de la mesure établie par la dimension de son propre corps. Plus que tout autre artiste, l'architecte est celui qui teste le passage à la réalité, la réalisation de l'échelle, la matérialisation de la structure, la puissance pragmatique du format. L'architecte met en œuvre la réalisation de la perfection abstraite, et vit pour le moment où l'objet quitte les deux dimensions du plan pour se matérialiser dans la réalité. Etienne-Louis Boullée (1728-1799) n'eut jamais l'heur de contempler ses projets à l'échelle, car ses plus brillants rêves demeurent couchés sur le plan - architecte visionnaire, il fut (comme ses collègues Ledoux et Lequeu) condamné à la fiction. De la même façon, Stourley Kracklite, l'Américain imaginé par Greenaway, ne pourra jamais réaliser le projet d'exposition destiné à rendre hommage à Boullée, et passe son temps parmi des maquettes de monuments jamais réalisés (Rome est également un "atelier » jonché de fragments antiques). Kracklite échoue précisément à ressentir en ses entrailles le génie du lieu italien, et tombe malade, somatisant son échec à ancrer son propre corps dans l'architecture du lieu. Il déambule dans un espace (Rome) qui n'est pas en synergie avec le temps (les siècles passés et leurs prouesses architecturales l'obsèdent). Kracklite invente alors de nouvelles mesures (les «boullées »), fantasme sur des formats qu'il juge parfaits, réfléchit à la manière de Walter Benjamin sur la reproductibilité de l'œuvre d'art - de la maquette à l'échelle 1 , de la rotondité de son propre ventre à sa reproduction aplatie en A 3 qui sort de la photocopieuse, etc. Kracklite digère littéralement le format en son ventre malade.

12 Analysant la puissance de la projection du format, Elie During plaide pour les architectes qui modélisent le monde en le dupliquant à l'échelle réduite par le biais de maquettes : le monde, dit-il, "doit être maquettisé » (23). Au-delà de sa dimension ludique et de sa revendication extravagante, cette assertion nous renvoie à la question théorique de premier plan qui la sous-tend : les maquettes jouissent dans notre univers culturel d'un statut incertain qui les apparente aux œuvres d'art. C'est ainsi que selon During, « la maquette contribue à l'instauration - dirait Souriau - de l'objet qu'elle maquettise " (27). Tout comme l'avènement du portrait en peinture, dont Victor Stoïchita nous apprend qu'il se double d'une réflexion sur la nature du tableau en tant qu'objet d'art, la maquette propose une modélisation qui questionne sur sa propre existence au sein du monde qu'elle serait censée représenter. La maquette fonctionne comme un super-dispositif, qui introduit la possibilité de la fiction dans l'acte de modéliser le monde qu'elle effectue : 
L'opération de réduction dimensionnelle, la sélection qu'elle suppose, n'excluent pas, d'ailleurs, que ces dimensions fonctionnent elles-mêmes dans une relation complexe à une pluralité d'échelles ou d'espaces de référence. Tout l'intérêt de la maquette, comme instrument de conception architecturale, est que l'objet référent n'existe pas encore, ou n'existe qu'en tant que projeté, ce qui autorise de fait « un ensemble de références possibles diverses là ou la correspondance de la carte au territoire ou de l'image au référent est univoque ». (31)

13 La maquette sort ainsi du strict rapport analogique (isomorphique) de correspondance qu'elle entretient avec le réel, et fonctionne à des échelles multiples, se pliant souplement à de multiples systèmes de référence, ou superposant plusieurs perspectives. La maquette ouvre l'espace de représentation en proposant des schématisations à l'observateur, et ainsi, on peut l'envisager comme ne renvoyant à rien d'autre qu'elle-même; la maquette devient alors prototype, et autorise une modélisation auto-suffisante, car sans référent. C'est ainsi que les architectes Boullée, Ledoux et Lequeu ont conceptualisé des architectures jamais réalisées de leur vivant, ce qui n'enlève rien à leur génie architectural visionnaire. ${ }^{4}$ Le grand avantage du modèle réduit, c'est qu'il offre la possibilité de la maîtrise de la totalité d'une forme, qui demeure contrôlable. La forme de la maquette, de par son format, est ainsi manipulable aussi bien par la main de l'homme que par sa pensée. Lévi-Strauss remarque que la majorité des œuvres d'art sont des modèles réduits, et écrit :

[le modèle réduit] n'est pas une simple projection, un homologue passif de l'objet ; il constitue une véritable expérience sur l'objet. [...] Par la seule contemplation, le spectateur est, si l'on peut dire, envoyé en possession d'autres modalités possibles de la même œuvre, et dont il se sent confusément créateur à meilleur titre que le créateur lui-même qui les a abandonnées en les excluant de sa création; et ces modalités forment autant de perspectives supplémentaires, ouvertes sur l'œuvre actualisée. Autrement dit, la vertu intrinsèque du modèle réduit est qu'il compense la renonciation à des dimensions sensibles par l'acquisition de dimensions intelligibles. (1960, 35-36)

On comprend bien comment cette modélisation du réel plaît à Greenaway, qui est régulièrement accusé de négliger, voire d'oblitérer le sensible et l'affect au profit de l'intelligible; on se souvient que ses réalisations explorent régulièrement les différents régimes d'exposition du monde, l'art lui fournissant la possibilité de faire apparaître des propriétés communes à la fois au signe et à l'objet, et tout particulièrement une structure qui se révèle dans la (re)présentation, qu'elle soit plastique ou textuelle (que l'on songe à son exposition Some Organising Principles en 1993, dans laquelle les artefacts étaient présentés sur leur envers, à la manière dont la nature morte opérait avant qu'elle ne fut instaurée en tableau (cf. Stoïchita, 1999). Claude Lévi-Strauss souligne que l'art aspire à la condition du langage, « il reconstruit le réel en le ramenant à des signes opérables, autrement dit à des systèmes d'équivalence entre des valeurs intellectuelles et sensibles, des séries d'objets hétérogènes. » $(1961,151)$. La modélisation artistique du monde permet ainsi de le rendre intelligible et plastiquement signifiant. La maquette, dont During nous dit qu'elle agit comme un instrument d'optique, prisme ou loupe grossissante, est un dispositif efficace agissant à de multiples niveaux sur la connaissance du monde: dispositif de capture, "elle extrait du réel les dimensions suffisantes qui permettent de transformer un sujet, tel qu'il soit, en objet d'expérimentation » (40). ${ }^{5}$ La fiction artistique est célébrée précisément parce qu'elle a le pouvoir d'intensifier l'expérience que le spectateur a du réel, rendant cette expérience plus vive, plus éclatante, plus vibrante que la vie. Tout se passe, souligne Richard Shusterman, « comme si la déviation de la réalité ordinaire, par la mise entre 
parenthèses de l'art, nous permettait d'emprunter une route indirecte pour apprécier le réel de façon bien plus pleine ou profonde " (163). L'architecture, même jamais réalisée à l'échelle 1, est donc un format à l'efficacité heuristique considérable.

Les expositions de la série The Stairs, ainsi qu'une grande partie des expositions récentes dont Greenaway fut commissaire, s'organisent précisément autour du thème de la puissance de l'opération de formatage généré par l'architecture. Il confie ainsi que l'escalier de la bibliothèque Lorenziana construit par Michel-Ange à Florence fut le prétexte à réaliser Prospero's Books, et que The Baby of Mâcon lui donna l'occasion de réfléchir à l'espace architectural de la procession, retravaillant ainsi les processions vénitiennes de Bellini et les Triomphes de César de Mantegna. ${ }^{6}$ Très souvent, c'est un élément architectural qui crée l'étincelle donnant naissance à une œuvre plastique, qu'elle appartienne au domaine des images mouvantes ou fixes. La question qui se pose alors est la suivante: Greenaway aurait-il donc fait des moyens ou longs-métrages au lieu de faire des installations ? Ou serait-ce le contraire, aurait-il fait des installations à défaut de faire des films ? La genèse compliquée de The Stairs tend à confirmer cette hypothèse.

\section{The Stairs, ou « l'artialisation » du monde par le dispositif}

Le format informe, au sens où il donne forme à. Dans son ouvrage Art et anticipation, le philosophe Alain Roger reprend le concept d'artialisation de Michel de Montaigne, et invoque une opération de médiation de la perception :

[...] Notre expérience, perceptive ou non, est "artialisée », c'est-à-dire modelée et donc anticipée par des modèles, médiateurs ou opérateurs artistiques, comme on voudra les appeler. [...] La première opération consiste à inscrire directement le code artistique dans la matérialité du lieu. On artialise in situ. C'est l'art millénaire des jardins, et, depuis le XVIII ${ }^{\mathrm{e}}$ siècle, celui des jardins paysages. L'autre manière est indirecte. On n'artialise plus in situ, on agit sur le regard, on lui fournit des modèles de vision, des schèmes de perception et de délectation, on artialise in visu, on anticipe la perception [...] $(1997 \mathrm{a}, 9)$

17 Dans Le Ventre de l'architecte, l'architecture offre ainsi le meilleur exemple d'art in situ. Souvenons-nous de l'aphorisme d'Etienne-Louis Boullée, gravé sur les murs de l'exposition de Kracklite à sa gloire : «l'architecture, c'est mettre en oeuvre la nature », ou sa variante " l'architecte doit être le metteur en oeuvre de la nature ${ }^{7}$ qui présuppose que la nature nécessite d'être mise en œuvre, d'être mise en ordre, d'être sublimée afin d'être perçue et estimée à sa juste valeur par les humains.

18 Faire de l'art in situ, au sens où Roger l'entend, est ainsi précisément ce que Stourley Kracklite et Etienne-Louis Boullée s'efforcent de mettre en oeuvre. Leurs projets sont en fait de l'art in visu qui ne parvient jamais à accéder au in situ. Boullée n'est jamais parvenu à faire construire aucun de ses projets les plus majestueux, et Kracklite ne parviendra jamais à terminer l'exposition qu'il a entrepris de monter à Rome, en dépit de ses efforts et à cause de la maladie qui ronge ses entrailles. La structure diégétique du long-métrage s'articule ainsi autour des difficultés liées au montage d'une exposition internationale. De façon très ironique, le projet The Stairs, qui fut initialement pensé comme un film par Greenaway, ne vit jamais le jour ; c'est ainsi qu'un film sur la genèse d'une exposition enfanta une exposition sur l'art de faire des 
films, ou tout du moins sur les caractéristiques du format des images fixes et mouvantes.

The Stairs offre une suite de points de vue au spectateur, construits au moyen de dispositifs qui cadrent, délimitent, norment, et formatent. La découverte de la perspective italienne à la Renaissance fut sans doute le format le plus mémorable de tous, et Greenaway se donne pour tâche de le questionner, tout en le renforçant et en le soulignant. Ainsi il choisit d'installer des cadres géants, à la mesure des corps humains qui en graviront les marches :

The post-Renaissance European classical tradition has viewed landscape primarily according to notions of perspective, symmetry and a desire to explain rationally an urban landscape space [...] Any venerable city is an amalgam of the secular and the ecclesiastical, the domestic and the industrial, wet and dry, open and closed, void and mass, the significant and the superficial. This selection of viewpoints pursued this variety, and did not neglect the denigrated viewpoint of the tourist, for the tourist seeks the definitive view, the economic identifying viewpoint with the maximum amount of sensory gratification. (Greenaway, 1994, 85)

Initialement, The Stairs (imaginée dès 1992) devait être un long-métrage tourné à Rome, tout comme The Belly of an Architect, mais Greenaway le concevait comme beaucoup plus dépouillé. Il s'agissait de rendre hommage à l'art de l'escalier, et au rôle central de l'élévation dans la perception du paysage, comme le révèle le jeu de mot souligné par son auteur entre stairs et stares. Le film devait raconter la fascination d'un peintre pour le $\mathrm{XVII}^{\mathrm{e}}$ siècle, à qui on commande une fresque. La réalisation de cette fresque devait attirer l'attention sur la question de la représentation et de la mimesis, spécialement sur la technique baroque du trompe-l'œil, le tout étant utilisé comme la métaphore du cinéma lui-même. Pour reprendre les termes de Greenaway, c'était une sorte de spectacle complet réunissant tous les moyens possibles et en particulier la lumière, pour créer l'illusion et prêcher un acte de foi et un «besoin de croire " essentiels à l'Eglise comme au cinéma (Greenaway, 1994, 39). Le film devait donc dénoncer la mégalomanie cinématographique et analyser les rouages par lesquels les images mouvantes parviennent à instaurer l'illusion, comparant ainsi indirectement images fixes et images mouvantes. Cependant, le projet de Greenaway ne vit jamais le jour, car:

Le film exigeait quelque chose de plus grand et de plus complexe que $2 \mathrm{~h}$ d'images asservies aux pratiques qui régissent le cinéma traditionnel. Trois écrans et une durée de 24 heures semblaient nécessaires pour rassembler de façon satisfaisante les idées telles que je les avais imaginées. C'était impossible. Qui financerait un tel film ? Comment le distribuer et qui accepterait de faire cette distribution ? Qui aurait même envie de le voir? (Greenaway, 1994, 40)

21 Ces problèmes avaient déjà été pressentis par Greenaway lors de la réalisation de The Belly of an Architect, premier volet du projet, et c'est pourquoi le réalisateur finit par adopter la solution de son personnage Kracklite : faire une exposition, faute de pouvoir recréer comme il en rêve les bâtiments dessinés par Boullée à l'échelle 1. The Stairs était donc une série de dix expositions dont chacune développerait une partie du problème théorique posé par le format cinématographique, à savoir, comprendre les limites de l'expérience vécue par le spectateur. Chaque exposition devait développer un aspect propre à la constitution de l'illusion, de manière à faire réfléchir le spectateur aux formats: le lieu, le public, le cadre, le jeu d'acteur, la lumière, le texte, le temps, l'échelle, les accessoires pour citer les principaux. 

financiers pour les mettre en œuvre: La première eut lieu à Genève sur le cadre en 1994, la seconde à Munich sur la projection en 1995, la troisième à Nagoya sur le costume. Trieste et Budapest devaient figurer parmi les villes suivantes, mais le coût des expositions se mit à dépasser celui du film initial, donc tout s'arrêta. Greenaway fut entre temps occupé par le tournage de The Cook, the Thief, His Wife and her Lover. L'espace urbain fut donc choisi comme le lieu idéal d'une théâtralisation pédagogique, réflexion sur le format, réalisation conçue pour donner à l'observateur le rôle non d'un simple œil, mais celui d'un acteur. L'œil reçut ainsi un corps pour le compléter, corps multiple du public invité à contempler de nouvelles perspectives à travers les dispositifs spéculaires dont Greenaway parsème la ville.

\section{Discipliner la perception : le rectangle et la mémoire}

The Belly of an Architect contient les germes qui feront éclore The Stairs. On se souvient que Kracklite, avant de se suicider, regarde dans une des nombreuses "boîtes de Canaletto" installée sur le toit du musée Vittoriano à Rome. Ces dispositifs de cadrages sont les versions modernisées des camera obscuras des siècles passés, et rappellent plus précisément les chambres optiques utilisées aux XVIII ${ }^{\text {e }}$ siècle par les peintres védutistes; Canaletto réalisait ses vues panoramiques au moyen de cet instrument, qui lui permettait de réinventer le genre du paysage en rendant hommage à l'architecture. Le rectangle de cadrage est lui aussi le dispositif choisi pour The Stairs, et le symbole de l'arbitraire du format choisi au cours de l'histoire par quantité de dispositifs optiques :

The example of Renaissance painting gave the frame to the stage, so that our perception of ballet, opera and theatre is almost exclusively of a performance inside and outside a rectangular proscenium arch; it gave the notion of a rectangular frame to the photograph, to cinema and to television in a historical sequence that continued to perpetuate four rightangles and four straight sides. Despite numerous Baroque and Rococo practitioners investigating non-regular shapes, the size and proportion of the painting frame in the last four hundred years have generally remained conservative, centreing [sic] around the 1 to 1.66 ratio used in the wide cinema screen, though this particular means is often up-ended to make the vertical proportion larger, to suit the needs of portrait-painters executing fulllength portraits. This proportion may, no doubt, be related to the golden section, a harmonious proportion arrived at by those with no special training in mathematics and no special knowledge or good taste or " eye ». (Greenaway, 1995, 19)

Les chambres optiques de The Stairs Genève sont également les pendants immaculés de la mire noire qui est l'instrument favori du peintre Neville dans The Draughtsman's Contract. Greenaway choisit pour sa première exposition à Genève de doter la ville de cent chambres optiques similaires aux boîtes de Canaletto de Kracklite, qui resteront à la disposition du public pendant cent jours afin que la ville lui devienne plus intelligible, et que l'opération de cadrage lui devienne plus apparente. Chaque dispositif est un escalier de bois peint en blanc mat. Chaque spectateur doit effectivement faire l'effort de monter l'escalier, passage obligé pour se retrouver en position élevée - si modeste soit-elle - et donc reconnaitre « la relation du projet avec l'escalier » ("acknowledge the stair-consciousness of the project", Greenaway, 1994, 82). Cet escalier n'a peut-être que quatre marches, peut-être dix, mais il faut faire un effort conscient pour contempler ce lieu à travers le cadrage. Les escaliers ont été érigés dans des parcs, dans de grandes artères ou de modestes allées, à des carrefours, dans des cours, dans des musées, regardant l'herbe, la pierre, le béton, le ciel, le marbre, la 
brique. Et contrairement au bref aperçu des lieux qu'offre le cinéma, dans ce film imaginaire les cent lieux sont disponibles vingt-quatre heures sur vingt-quatre pendant cent jours, formant réseau cognitif: «Many framings are devised to be quotations of stairness, to make a deliberate choice of composition that will reflexively frame a staircase. And in the spirit of sequence that governs the construction of a film however minimally narrative - there is a constant concern to make as many multiple connections between viewpoints as possible. » (Greenaway, 1994, 85)

Greenaway se plaît à désigner l'exposition de Genève comme «a 100-viewpoint film without a camera", qui contient tous les ingrédients d'un film mais restera la projection individuelle de chacun. Greenaway se souvient avec amusement des mots d'un enfant s'exclamant en haut des marches : « Mais il n'y a rien à voir ! ${ }^{8} \mathrm{~A}$ Munich en octobre, l'artiste fait de manière similaire dresser cent écrans de projections à travers la ville, fonctionnant de $6 \mathrm{~h}$ du matin à minuit. J'ai déjà évoqué ailleurs le problème de l'asservissement du cinéma à la narration (cf. Gasquet, 2005, 2015), mais Greenaway a également d'autres chevaux de bataille, à savoir l'uniformisation des conditions de visionnage, et la répétition formelle inéluctable et lassante (Cf. Gasquet 2010). Le cinéma nous donne à voir toujours la même forme, déclinée avec des variations minimales. C'est également de cela que souhaite faire prendre conscience le réalisateur en offrant au spectateur des cadres peut-être inutiles. Le cinéma n'est qu'un médium qui parvient à faire fonctionner ensemble une conglomération d'autre média ; son succès tient à sa capacité d'être diffusé en masse sans perte de qualité (cf. Greenaway, 1994, 4).

Le format rectangulaire est omniprésent depuis plusieurs siècles, et ce n'est certainement pas le fruit du hasard si l'architecte Kracklite choisit d'exposer Boullée dans le célèbre monument Vittorio-Emmanuele à Rome. Ce palais est surnommé par les habitants de la ville la " machine à écrire » (en italien " macchina da scrivere ») de par sa ressemblance avec la célèbre Olivetti M40. Le papier fut le format autour duquel s'organisa la plus grande normalisation en Occident, comme le souligne Le Corbusier dans L'Art décoratif aujourd'hui :

Dès que la machine à écrire est née, le papier à lettres fut standardisé : cette standardisation eut une répercussion mobilière considérable, conséquence de l'établissement d'un module, celui du journal commercial. Les machines à écrire, les copies de lettres, les corbeilles à classement, les dossiers, les tiroirs à dossier, les meubles à classement, en un mot toute une industrie mobilière, fut conditionnée par l'établissement de ce standard [...] Le format commercial n'est pas une mesure arbitraire. Qu'on apprécie plutôt la sagesse (moyenne anthropocentrique) qui l'a établi. De toutes ces choses d'usage universel, la fantaisie individuelle s'incline devant le fait humain (16).

C'est précisément ce qui terrasse Kracklite, qui perd la raison, obsédé par la standardisation dans le long-métrage. Il cherche désespérément à mesurer, à reproduire les contours de son propre corps à l'aide des machines qui ont été bâties pour un format spécifique, mais il est bien obligé de constater qu'il est hors-format... il n'a pas sa place dans le réseau normatif qu'est devenu le monde. Le standard se déploie ainsi progressivement, presque insidieusement, par involutions diégétiques successives, et par étapes sur le corps de Kracklite qui ne peut rentrer dans le cadre européen qu'il a pourtant étudié de façon abstraite toute sa vie.

La question du format recoupe aussi celle de la modélisation opérée par la mémoire. The Belly of an Architect interroge également le format mémoriel par le biais de la 
représentation dévolue aux cartes postales, elles aussi fondées sur le format rectangulaire. L'humain acquiert par le biais de ces images populaires formatées une vision parcellaire du paysage, et Greenaway se demande ce que ces représentations induisent, et ce que leur juxtaposition peut retenir, voire créer dans la représentation formatée de l'architecture d'une ville. Ainsi, la moitié du script publié chez Faber est consacrée aux 124 cartes postales que le malheureux Kracklite envoie régulièrement à son ami imaginaire Boullée. A l'origine, Greenaway souhaitait que l'intégralité des cartes postales invoquées composent un court métrage indépendant qui aurait été projeté à la suite du long-métrage originel, sous le nom de Dear Boullée. Le fantôme muet de Boullée devient ainsi le confident de Kracklite ; la scène initiale où l'architecte de Chicago explique la raison de cette correspondance unilatérale fut coupée au montage. On y voyait Kracklite expliquer au petit garçon qui regarde avec lui les ébats adultères de sa femme par le trou d'une serrure que Rome est la seule ville au monde dont l'apparence totale peut être reconstituée au moyen de cartes postales. Chaque " point de vue », devenu tel parce qu'inlassablement reproduit, fait en effet écho à un autre, en raison de la multiplicité exceptionnelle des monuments et des endroits architecturalement signifiants. C'est pourquoi Kracklite pense naïvement que grâce à cette série de cartes postales, son ami imaginaire Boullée pourra créer sa propre carte de Rome depuis Paris (parce que Boullée "vit à Paris". cf Greenaway, 1988, 73-74). Cette reconstitution de la Ville Eternelle par le biais de points de vue juxtaposés de force, jurant les uns avec les autres, constitue ici une fascinante représentation : la variété des perspectives en souligne précisément toute l'artificialité, et il est bien entendu ironique que ces visions bigarrées soient couchées sur papier par le biais de la photographie, associée par le grand public à la « réalité » de par son isomorphisme. La mosaïque de cartes postales aux couleurs ultra-saturées de notre architecte donne donc paradoxalement à voir sa propre facticité ; la photo n'offre qu'un moyen supplémentaire de représenter le réel, après les plans, après la peinture. Il serait hérétique de la penser plus fidèle que les autres médias visuels.

\section{Conclusion : le dispositif grandeur nature, ou l'architecture comme « performance de l'histoire »}

Les villes sont selon Greenaway à concevoir comme des théâtres de la mémoire avec leur architecture en toile de fond (Cf. Camillo, Yates). Une célèbre scène du Ventre de l'Architecte nous présente la fine fleur du monde de l'art applaudissant le Panthéon de Rome : sur le plateau de tournage, les techniciens se mirent également à applaudir, tout comme le public. Greenaway souligne que ces applaudissements n'étaient pas vains :

The applause was not wasted. Maybe we were applauding the building's distant author or its long dead patron. Maybe the applause was for the building's history, its vicissitudes or its magnificent decay. Maybe we were exhilarated to be clapping such a particular mixture of stone and shadow. Maybe the applause was to express a sheer exuberant delight in the canons of exhilarating architecture, in an exciting use of place and indeed in the performance of history that has both made and swept around that building. We were undoubtedly clapping a performance, a performance perhaps of History and Time and Sense of Place, though that performance was not conventionally "alive" or rehearsed or artificially provided for us as an officially attentive audience. But then, why should it be? (Greenaway, 1994, 91) 
Ainsi, de même que dans les jardins d'André Le Nôtre les humains devenaient les fleurs que l'architecte des jardins omettait volontairement de ses parterres végétaux, dans une ville, nous sommes nous aussi des acteurs sur un plateau dont nous ne lasserions jamais ; si le spectacle, si le tableau est réussi, alors nous ne devrions jamais vouloir le quitter. L'excellence de la mise en scène de l'espace urbain se mesure ainsi à la difficulté éprouvée à le quitter ; l'architecture donne à la ville sa forme de représentation. C'est pour Greenaway ce qui se produit souvent en Italie, où l'excitation visuelle est à son comble. Cependant, peut-être que les meilleurs architectes resteront au fond les architectes de papier, comme Boullée ou Ledoux, les visionnaires n'ayant jamais eu à se confronter aux trois dimensions: pas besoin de se préoccuper des évacuations, des toilettes et des parkings, comme dit Greenaway. Ce dernier confie que ce n'est que tardivement qu'il a réalisé que très peu d'architectes construisent, et que la plupart se contentent de dessiner et de rêver à la perfection : «An ideal situation. To look and walk about and think about architecture and architectural spaces without any responsibility whatsoever; with as much word-play as you want. Ideally you should not walk [...] but you should glide about on a camera dolly with a crane-hoist possibility. Nothing better. You can entirely concentrate on space and at a regular and steady pace. » (Greenaway, 2010, 40). Le travelling autoriserait ainsi l'esprit à jouir du format architectural glissant devant l'œil du spectateur, introduisant une souplesse à l'intérieur du cadre de projection, épousant ainsi la fluidité du temps et de la perception. Les modes de représentation que nous avons étudiés ici par le biais des œuvres de Greenaway, rendant compte de l'omniprésence du format et du formatage, confirment que l'établissement d'une représentation fictionnelle, sémiotisée et reconnue comme telle par le biais de dispositifs, acte ainsi le processus de fictionalisation décrit par Jacques Rancière dans le Partage du Sensible :

Le réel doit être fictionné pour être pensé. [...] Il ne s'agit pas de dire que tout est fiction. Il s'agit de constater que la fiction de l'age esthétique a défini des modèles de connexion entre présentation de fait et raison de la fiction, et que ces modes de connexion ont été repris par les historiens et par les analystes de la réalité sociale. Écrire l'histoire et écrire des histoires relèvent d'un même régime de vérité. Cela n'a rien à voir avec aucune thèse de réalité ou d'irréalité des choses. » (2000, 60-61)

31 Ce que Rancière nomme partage du sensible, c'est « la façon dont les formes d'inclusion et d'exclusion qui définissent la participation à une vie commune sont [...] configurées au sein même de l'expérience sensible de la vie " (Rancière, in Palmieri, 2002). Cette configuration, on le comprend, passe par la constitution de formats qui modéliseront l'expérience et lui permettront de s'ancrer dans le réel, d'être perçue, désignée, saisie et mesurée, en même temps qu'elle saisira dans un double mouvement le réel, le percevra, le désignera, le saisira, le mesurera. Michel Foucault, lorsqu'il dresse l'état des lieux du monde, dresse la carte des formatages de la pensée humaine qu'il classe selon des épistémê repérables précisément grâce aux changements des formats qui caractérise nos artefacts (écrits, objets d'arts, théorèmes scientifiques - in Foucault, 1966).

32 Notre pensée se coule ainsi dans le format, en même temps que nos catégories épistémologiques s'arc-boutent sur lui, et le constituent tout en le représentant dans un mouvement autoréflexif fascinant. L'architecture selon Greenaway symbolise parfaitement cette capacité involutive et réflexive du savoir humain, ce métamodèle qui permet de voir le dedans et le dehors, et d'être aussi vu du dedans et du dehors; ce que je nomme métamodèle ici est ce qui a été décrit comme "hétérotopie » par 
Foucault (1967). L'architecture met donc bien en cuvre la nature au sens où l'entendait Louis-Etienne Boullée, et elle établit également un formatage des plus importants, puisqu'elle consacre dans son esthétique la toute-puissance du savoir tout en instaurant le pouvoir de l'homme sur la nature. Nelson Goodman nous a révélé dans Ways of Worldmaking que nous fabriquons de multiples versions du monde au moyen de symboles que nous disséminons partout autour de nous ; ainsi, le format, par le biais de ses multiples modalités (accentuation, atomisation, déformation, répétition, traduction) et métamodèles, fabrique des mondes en restructurant sans arrêt les formes et les contenus mêmes de l'accès à la réalité. Nous apprenons à percevoir, à voir, et à savoir au sein de l'écheveau inextricable de l'expérience; le format est le fil qui constitue cet écheveau.

\section{BIBLIOGRAPHIE}

\section{Ouvrages cités}

AGAMBEN, Giorgio. Qu'est-ce qu'un dispositif ? 2006. Trad. Thomas Rueff. Paris : Payot, 2007.

ALBERTI, Leon Battista. Della Pittura, La Peinture. 1435. Trad. Thomas Golsenne et Bertrand Prévost. Paris : Seuil, 2004.

BARIDON, Michel. Le Jardin paysager anglais au XVIII siècle. Dijon : EUD, 2000.

BARIDON, Michel. Les Jardins. Paysagistes, jardiniers, poètes. Paris : Robert Laffont, 1998.

BENJAMIN, Walter. L'Euvre d'art à l'ère de sa reproductibilité technique. 1939. Paris : Allia, 2012.

BOULLEE, Etienne-Louis. Architecture. Essai sur l'art. 1768-1793. Paris : Hermann, 1968.

CAMILLO, Giulio. Le Théâtre de la mémoire. Paris : Allia, 2001.

CAUQUELIN, Anne. L'Invention du paysage. Paris : PUF, 2000.

CORBIN, Alain. L'Homme dans le paysage. Paris : Textuel, 2001.

CRARY, Jonathan. Techniques of the Observer, on Vision and Modernity in the Nineteenth Century. Cambridge, Mass. : MIT publications, 1992.

DAMISH, Hubert. L'Origine de la perspective. Paris : Champs Flammarion, 1987.

DELEUZE, Gilles. « Qu'est-ce qu'un dispositif ?» Michel Foucault philosophe. Paris : Seuil, 1989.

DURING, Elie. « Le monde doit être maquettisé » in David Zerbib, ed. In Octavo : des formats de l'art. Dijon et Annecy : Les Presses du Réel : ESAAA, 2015. 23-43.

EHRARD, Jean. L'Idée de nature en France dans la première moitié du XVIII ${ }^{e}$ siècle. Paris : Albin Michel, 1994.

ELLIOTT, Bridget, PURDY, Anthony. Peter Greenaway. Architecture and Allegory. Chichester: Academy editions, 1997. 
FEL, Loïc. L'esthétique verte : de la présentation à la représentation de la nature. Seyssel : Champ Vallon, 2009.

FOCILLON, Henri. Vie des formes. Paris : PUF, 1934.

FOUCAULT, Michel. Les Mots et les choses. Paris : Gallimard, 1966.

FOUCAULT, Michel. « Le Jeu de Michel Foucault ». 1977. Dits et écrits, II. Paris : Gallimard, 1994.

FOUCAULT, Michel. « Des espaces autres ». 1967. Dits et écrits, II, 1984 (conférence au Cercle d'études architecturales), in Architecture, Mouvement, Continuité, ( ${ }^{\circ} 5$, octobre 1984) : 46-49.

GASQUET, Lawrence. « Peter Greenaway et la question de la primauté de l'image ou du texte » in Texte / Image : nouveaux problèmes, in Liliane Louvel et Henri Scepi, eds. Rennes : Colloque de Cerisy / P.U.R., 2005.

GASQUET, Lawrence. «Miles and miles and miles of painted darkness: Peter Greenaway and the Intelligibility of Representation », Revue Cycnos, numéro Special Peter Greenaway, 26 (2010) : 99-115.

GASQUET, Lawrence. « Peter Greenaway's Expanded Cinema : quelques réflexions sur l'intermédialité. » In Raphaël Jaudon, Dario Marchiori et Luc Vancheri, eds. Ecrans : Expanded Cinema. Paris : Classiques Garnier, (2015-1, $\left.n^{\circ} 3\right)$.

GOODMAN, Nelson. Ways of Worldmaking. Indianapolis: Hackett, 1978.

GRAS, Vernon \& Marguerite. ed., Peter Greenaway Interviews. Jackson: University Press of Mississipi, 2000.

GREENAWAY, Peter. Some Organising Principles. Swansea: Glynn Vivian Gallery, Great Britain, 1993. GREENAWAY, Peter. The Belly of an Architect. Londres : Faber \& Faber, 1988.

GREENAWAY, Peter. The Stairs 1, Geneva The Location. Londres : Merell Holberton, 1994.

GREENAWAY, Peter. The Stairs 2, Munich Projection. Londres : Merell Holberton, 1995.

GREENAWAY, Peter. « Utopian Cities » in L'Italia delle Citta, Italy of the Cities. Milano: Skira, 2010.

HUNT, John Dixon. Greater Perfection: The Practice of Garden Theory. Londres : Thames \& Hudson, 2000.

KEESEY, Douglas. The Films of Peter Greenaway, Sex, Death and Provocation. Jefferson: Mc Farland and Co, 2006.

LAWRENCE, Amy. The Films of Peter Greenaway. Cambridge : Cambridge University Press, 1997.

LE CORBUSIER. L'Art décoratif aujourd'hui. Paris : Crès, 1925.

LEMAGNY, Jean-Claude. Boullée, Ledoux, Lequeu, Visionary Architects. Houston: University of St Thomas, 1968.

LEVI-STRAUSS, Claude. La Pensée sauvage. Paris : Plon, 1960.

LEVI-STRAUSS, Claude. Entretiens avec Georges Charbonnier, Paris : Julliard, 1961.

MARTINET, Marie-Madeleine. Art \& Nature en Grande-Bretagne au XVIII siècle, de l'harmonie classique au pittoresque du premier romantisme, $17^{e}-18^{e}$ siècles. Paris : Aubier Montaigne, 1980.

PALMIERI, Christine. « Compte rendu et interview de Jacques Rancière, Le partage du sensible ». ETC (2002 -59) : 34-40.

PREVOST, Bertrand. La Peinture en Actes. Paris : Actes Sud, 2007. 
RANCIERE, Jacques. Le Partage du sensible : esthétique et politique, Paris : La Fabrique Editions, 2000.

ROGER, Alain. Art et Anticipation. Paris : Editions Carré, 1997a.

ROGER, Alain. Court traité du paysage. Paris : Gallimard, 1997b.

SHUSTERMAN, Richard. « Pourquoi dramatiser ? L'art et son cadre » in Zerbib, David, ed, In Octavo : des formats de l'art. Dijon et Annecy : Les Presses du Réel / ESAAA, 2015. 149-167.

SOURIAU, Etienne. Les Différents modes d'existence. Paris : PUF, 2009.

STOICHITA, Victor. L'Instauration du tableau. Genève : Droz, 1999.

THIBERGIEN, Gilles. Nature, Art, Paysage. Paris : Actes Sud, 2001.

WOODS, Alan. Being Naked Playing Dead. Manchester: Manchester UP, 1996.

YATES, Frances. The Art of Memory. 1966. Londres : The Bodley Head, 2014.

ZERBIB, David. In Octavo : des formats de l'art. Dijon et Annecy : Les Presses du Réel / ESAAA, 2015.

\section{NOTES}

1. «D'abord, écrit-il, j'inscris sur la surface à peindre un quadrilatère à angles droits aussi grand qu'il me plait, qui est pour moi en vérité comme une fenêtre ouverte à partir de laquelle l'histoire représentée pourra être considérée ; puis j'y détermine la taille que je souhaite donner aux hommes dans la peinture. " Alberti, édition de Thomas Golsenne et Bertrand Prévost, 2004, 82-83. A ce sujet, voir aussi Prévost, 2007.

2. Michel Foucault nomme "dispositif » un espace institutionnel qui fonctionne sur le modèle de machines à faire voir et à faire parler. Cet espace met en œuvre des pratiques discursives et non discursives; le dispositif est le « réseau » qu'il est possible de tracer entre les différents constituants $d$ '« un ensemble résolument hétérogène, comportant des discours, des institutions, des aménagements architecturaux, des décisions réglementaires, des lois, des mesures administratives, des énoncés scientifiques, des propositions philosophiques, morales, philanthropiques, bref : du dit, aussi bien que du non-dit » (Foucault, 1977, 299). A ce sujet, voir aussi Deleuze, 1989, 185-195. Giorgio Agamben élargit la définition du dispositif comme étant « tout ce qui a, d'une manière ou d'une autre, la capacité de capturer, d'orienter, de déterminer, d'intercepter, de modeler, de contrôler et d'assurer les gestes, les conduites, les opinions et les discours des êtres vivants. » (Agamben, 2006).

3. A ce sujet, on peut consulter Greater Perfection: The Practice of Garden Theory de John Dixon Hunt, L'Invention du paysage d'Anne Cauquelin, Les Jardins. Paysagistes, jardiniers, poètes de Michel Baridon, L'Homme dans le paysage d'Alain Corbin, L'Origine de la perspective de Hubert Damish, L'esthétique verte : de la présentation à la représentation de la nature de Loïc Fel, Art \& Nature en Grande-Bretagne au XVIIIème siècle de Marie-Madeleine Martinet, Nature, Art, Paysage de Gilles Thibergien.

4. Voir Jean-Claude Lemagny, 1968.

5. Dans une perspective similaire, voir l'étude de Jonathan Crary sur la camera obscura comme format de la modernité, 1992.

6. «We did rebuild that staircase in wood and gesso and artificial marbling in a Rotterdam shipyard, and with no great regret we had to tear it down after two days to 
make way for another set. The film Prospero's Books was a vast Mannerist flourish with a shoestring budget. The fake marble columns were painted and repainted and used over and over again in different contexts. But then, it would be remembered in a completely different time-scale, that is what so many Italians cities do - Venice and Rome and Syracuse are famously full of re-used architectural fragments. » Greenaway, 2010, 57.

7. «Qu'est-ce que l'architecture ? La définirai-je avec Vitruve l'art de bâtir ? Non. Il y a dans cette définition une erreur grossière. Vitruve prend l'effet pour cause. Il faut concevoir pour effectuer. Nos premiers pères n'ont bâti leurs cabanes qu'après en avoir conçu l'image. C'est cette production de l'esprit, c'est cette création qui constitue l'architecture [...] Oui, je ne saurais trop le répéter, l'architecte doit être le metteur en oeuvre de la nature. » Boullée, 1768-1793, 73.

8. "Conceivably we have, therefore, the one hundred locations, the encapsulated metaphor, a commentary and the pictorial script. With these, there is material for a 100-day-long, 100-viewpoint film without a camera ». Greenaway, 1994, 95. Voir également Greenaway, 1995, 29.

\section{RÉSUMÉS}

Prenant le contre-pied de la célèbre formule d'Alberti qui en 1435 proposa d'utiliser le cadre d'une fenêtre afin de représenter le monde depuis le point de vue fixe d'un observateur, Peter Greenaway déclare que son travail n'est pas une fenêtre qui s'ouvre sur le monde, mais le résultat d'une composition effectuée au moyen de dispositifs. La représentation perspectiviste s'effectue par le biais d'un cadre qui contre toute attente ouvre le champ, et pose l'illusion de la profondeur comme convention, légitimant ainsi le format standardisé de la représentation picturale en Occident après la Renaissance. Le plasticien souligne toujours la facticité de la représentation et de ses formats. Ainsi, le format selon lui gagne toujours à être accentué, à être reconnu, voire à être dénoncé pour ce qu'il est. Greenaway aime à répéter qu'on n'échappe jamais à l'architecture : il conçoit les villes comme des théâtres de la mémoire qui s'organisent autour d'une architecture qui devient métamodèle, reflet de l'intellect humain et de ses formats de prédilection.

Taking arms against Leon Battista Alberti who in 1435 argued that a window frame was an ideal metaphor to describe the world as seen by an observer, Peter Greenaway insists that his work should not be seen as "a window on the world" but as "a self-conscious and artificial construct». Where Renaissance artists managed to produce the illusion of depth by rendering pictorial convention invisible - thanks to the universal adoption of the theory and practice of perspective - Greenaway chooses to foreground the artificial and arbitrary nature of the formats with which he works. He sees his role less as a filmmaker or visual artist than as an architect whose output is not a portrait of an external reality but the construction of a world that, while self-consciously artificial and therefore freed from dependence on the abandonment of disbelief, is capable of enveloping the viewer. Greenaway likes to say that architecture spares no one: he conceives cities as Memory Theatres which feature architecture functioning as a metamodel. For him, architecture is a symbolic construct representing the human intellect at play the world and, as such, a format that empowers mankind. 


\section{INDEX}

Keywords : Greenaway (Peter), architecture, perspective, photography, frame, model, standard, scale, construction, plasticity, epistemology

Mots-clés : Greenaway (Peter), architecture, perspective, photographie, cadre, maquette, échelle, construction, plasticité, épistémologie

\section{AUTEUR}

\section{LAWRENCE GASQUET}

Université Jean Moulin Lyon III

I.E.T.T., EA 4186

Lawrence Gasquet est Professeur à l'université Jean Moulin - Lyon III. Elle est spécialiste de l'oeuvre de Lewis Carroll, et travaille depuis de nombreuses années sur la théorie de l'art développée par John Ruskin, et sur les relations transesthétiques en général. Elle s'intéresse à l'histoire de la photographie, et tout particulièrement aux bouleversements épistémologiques que l'invention de la photographie a entraîné en France et en Angleterre au XIXe siècle. Elle étudie les affinités de ce médium avec l'art et la science. Sa recherche explore les relations intersémiotiques entre le texte et l'image, et interroge les diverses stratégies qui interviennent dans l'acte de représenter le réel ; elle étudie également l'histoire des musées à travers le prisme de la mise en scène visuelle des artefacts dans le monde occidental. Elle est l'auteur de Lewis Carroll et la persistance de l'image (Presses universitaires de Bordeaux, 2009). Elle a co-dirigé Lewis Carroll et les mythologies de l'enfance (Presses Universitaires de Rennes, 2005), L'Art de plaire (Gérard Monfort, 2006), L'Eblouissement de la Peinture, Ruskin sur Turner (Presses Universitaires de Pau, 2006). 